Review

\title{
Advances in the Regulatory Effects of Bioactive Peptides on Metabolic Signaling Pathways in Tumor Cells
}

\author{
Hongwei Cui*, Wenyan Han*, Junyao Zhang, Zhihui Zhang, Xiulan Su ${ }^{\bowtie}$ \\ Clinical Medical Research Center of the Affiliated Hospital, Inner Mongolia Medical University, Hohhot, 010050, Inner Mongolia, P.R. China \\ ${ }^{*}$ Co-first authors \\ $\triangle$ Corresponding author: Xiulan Su, Clinical Medical Research Center of the Affiliated Hospital of Inner Mongolia Medical University, Tongdao North Stress \\ No. 1, Hohhot, Inner Mongolia, 010050, People's Republic of China. Tel: +86 13904710692; Fax: +86 471 3451709; E-mail: xlsu@hotmail.com \\ (C) Ivyspring International Publisher. This is an open access article distributed under the terms of the Creative Commons Attribution (CC BY-NC) license \\ (https://creativecommons.org/licenses/by-nc/4.0/). See http://ivyspring.com/terms for full terms and conditions.
}

Received: 2018.11.08; Accepted: 2019.04.07; Published: 2019.05.26

\begin{abstract}
Changes in cell metabolism are an important feature of tumors that has always been an intense topic of study, particularly in regard to whether metabolic disorders are a cause or an effect of tumorigenesis. Studies have shown that the processes underlying metabolic changes in tumors involve the activation of protooncogenes and the inactivation of cancer suppressor genes, as well as changes in metabolic flux in cells due to the abnormal activation of signaling pathways that modulate metabolic enzymes and/or metabolic regulatory proteins at several levels, including transfer and posttranslational modification. Thus, the repair of abnormal metabolic pathways via intervention in the relevant tumor metabolic pathways that impact specific targets has become a new method of cancer prevention and treatment. Bioactive peptides, which have many biological functions, could specifically target malignant tumors. Their interaction with signal transduction molecules involved in the development and transference of tumors could regulate the relevant cell metabolic pathways and inhibit the development of tumors and/or accelerate apoptosis in tumor cells. In this review, several aspects of tumor suppression using bioactive peptides will be discussed and summarized, including the regulation of the PI3K/AKT/mTOR, AMPK, and STST3 signaling pathways, the modulation of the TRAIL death receptor signaling pathway, the regulation of aerobic glycolysis by PKM2, and the modulation of the NF-KB signaling pathway, to aid in the search for better and more specific antineoplastic drugs in the form of bioactive peptides.
\end{abstract}

Key words: bioactive peptide, tumor, cell metabolism, signaling pathway

\section{Introduction}

Changes in cell metabolism are an important feature of tumors in terms of tumorigenesis and the development of the tumor; whether these changes are a cause or an effect of tumorigenesis has always been a topic of intense study. It was first revealed by the Nobel Prize winner Otto Warburg in 1924 that compared to normal mature cells, tumor cells consume more glucose with greater efficiency to generate energy and support the need for rapid growth. Even when enough oxygen is present, the tumor cell degrades excess glucose it takes in via glycolysis, which produces a large amount of lactic acid, instead of using the process that involves the three carboxylic acid cycle and oxidative phosphorylation. This describes the famous "Warburg Effect" [1].
The only way for a tumor cell to acquire energy is via the degradation of glucose, which is an essential metabolic process in malignant tumors [2,3]. Both genetic and epigenetic factors have different levels of impact on various signaling pathways in cells. The carcinogenic signaling pathway would eventually alter the metabolic pathways, including those underlying glyco-metabolism, lipid metabolism, cholesterol metabolism, and iron metabolism. These changes in metabolism are an indispensable part of the process of tumorigenesis and the development of tumors [4]. Through these unusual metabolic pathways, tumor cells acquire the necessary materials and energy for nonstop proliferation; thus, research on the relevant metabolic pathways and mechanisms may improve 
the treatment of cancer by finding ways to intervene in signaling pathways in tumor cells, to inhibit cancer development and transference and, finally, to induce apoptosis in cancer cells [5].

Usually, bioactive peptides would have 2-20 amino acid residues, and their activity would vary depending upon the different kinds of amino acids they contained and their sequence. They have many biological functions, including anti-oxidation, antiproliferation, and the inhibition of tubulin polymerization and cytotoxicity. The functions of these peptides show that they have the potential to be used in cancer treatment [6-8]. There are various kinds of bioactive peptides that possess different biological functions and have close relationships with many regulation systems in the human body. Scholars in this field have already conducted many studies on various aspects of cancer, including its pathology, cellular molecules, genes and proteins, and the results have shown that bioactive peptides are preferable for use in the modulation of growth and metabolism in tumor cells. The peptides that have been studied include bioactive peptides that were extracted from the splenic organs of animals and spirulina [9-11]. There are several metabolic pathways in tumor cells: the PI3K/AKT/mTOR pathway, AMPK pathway, STAT pathway, various stress pathways (heat shock, DNA damage, p53, anaerobic, and p38/JNK pathways), inflammatory cytokine pathways (cox-2 and NF-KB pathways), survival pathways (NF-KB pathway), TNF-apoptotic pathway, and antiproliferation pathway (TGF- $\beta$ pathway) [12-17]. In this review, the main signaling pathways used by bioactive peptides to modulate the metabolism of tumor cells, including the PI3K/AKT/mTOR, AMPK, STAT3, TRAIL death receptor signal, and NF-KB signaling pathways, will be discussed and summarized. This review is expected to provide a reference for further studies on the mechanisms underlying the inhibition of the proliferation of tumor cells by bioactive peptides, as well as the development of antineoplastic foods and drugs.

\section{Regulation of the PI3/AKT/mTOR signaling pathway by bioactive peptides \\ PI3/AKT/mTOR signaling pathway}

The PI3K/AKT/mTOR signaling pathway, which is closely related to the growth and proliferation of cells, is an important pathway in cells [18]. The $\mathrm{PI} 3 \mathrm{~K} / \mathrm{AKT} / \mathrm{mTOR}$ signaling pathway in tumor cells is always activated, which increases their rates of growth and survival. There are three main ways to activate this pathway: 1) saltation of PI3KCA; 2) activation of receptor tyrosine kinase; 3 ) loss of PTEN activity, which reduces the decomposition of acid groups that are activated by PI3K in tumor cells [19]. Studies have shown that AKT, one of the downstream effector genes of PI3K, could stimulate tumor cells and switch their method glucose consumption to aerobic glycolysis. AKT, which is activated by PI3K, PDK1, and mTORC2, accelerates anaerobic respiretion and aerobic oxidative catabolism in cells after activation by upregulating the expression of glucose transporters (GLUT1, GLUT2, and GLUT4) and directing them to the cell membrane, which increases the uptake of glucose. Additionally, AKT1 can phosphorylate the key enzymes involved in glycolysis, including hexokinase and phosphofructokinase 2 [20]. In addition, usually only hypoxic conditions lead to the abundant expression of HIF1a, which would not be ubiquitinated and decomposed rapidly under in those circumstances. However, when $\mathrm{PI} 3 \mathrm{~K} / \mathrm{AKT} / \mathrm{mTORC} 1$ is activated, even when there is enough oxygen, the expression of HIF1a will be upregulated. As a translator, HIF1a could promote the expression of many key proteins involved in anaerobic respiration [21]. In addition, mTORC1 could promote the de novo synthesis of some lipids and the expression of key enzymes that interact with pentose phosphoric acid, which would contribute to the process of glycolysis in tumor cells and, in turn, promote proliferation and invasion [22].

Recently, researchers have found a new kind of polypeptide that is encoded by lncRNA LING00961 and is located in nucleus and lysosome that interacts with the lysosomal V types of ATP enzymes to inhibit the activity of mTORC1. This polypeptide is known as the small regulatory polypeptide of amino acid response (SPAR). Further studies have shown that when it is damaged, the lncRNA encoding SPAR would be inhibited when skeletal muscle is damaged; in the meantime, SPAR could lower the activity of mTORC1 and aid in the process of anathrepsis, which implies that the activity of mTORC1 may be modulated in a tissue in a specific way in response to damage and provides new evidence for the regulation of certain organized biological functions by the lncRNA-coded polypeptide [23].

$\mathrm{Li}$ and his colleagues have discovered a polypeptide fragment within the noncollagen NC1 domain of the collagen IVa3 chain that encodes a tumstatin with a relative molecular weight of $28 \mathrm{kDa}$ that contains 244 amino acid residues. When combined with integrin av $\beta$ on the surface of endotheliocytes independently of RGD, the polypeptide fragment is able to inhibit the phosphorylation of the tyrosine residues in FAK (focal adhesion kinase), which inhibits the activity of FAK and, in turn, leads to the suppression of PI3 kinase (phosphatidyl inositol 
3 kinase); as a result, the activity of PKB/Akt (protein kinase B) would be inhibited and endotheliocyte proliferation would be slowed. On the other hand, tumstatin is the inhibitor of the mTOR kinases and an essential protein for the process of cellular signal conduction; therefore, the phosphorylation of 4E-BP1 would be inhibited and the binding of 4E-BP1 and eIF4E would be strengthened, and this would result in the inhibition of HAT-dependent translation and the generation of endotheliocytes, which would thus promote their apoptosis [24]. As shown in Fig 1.

\section{Regulation of the AMPK signaling pathway by bioactive peptides}

AMPK (AMP-activated protein kinase) functions as a coordinator of the growth and metabolism of cells. By inhibiting mTORC1, AMPK can, in turn, regulate the activity of AKT; thus; AMPK is essential for metabolism regulation [25]. In tumor cells, some oncogenes and saltated antioncogenes inhibit the AMPK signaling pathway, which causes the growth of cells to no longer be regulated in step with energy metabolism and would lead to the survival of tumor cells even in abnormal nutritional conditions. For example, LKB1, an antioncogenic kinase that is required for the activation of AMPK, is usually saltated nonsmall cell cancer and cervical cancer [26]. After the loss of AMPK activity, AKT is able to activate mTOR and HIF1 [27].

The research of Wang [28] and Mo[29] has shown that the Hippo signaling pathway may be manipulated to regulate the metabolism of glucose. During this process, there are two key proteins, YAP and AMPK, that are involved in signal cascade events. YAP functions as a helper in the growth and transference of cancer cells. The researchers found that when there is insufficient glucose in the environment, the AMPK enzymes would be activated and cause the YAP protein to be deactivated. Additionally, the regulation of YAP can be achieved via partial manipulation of the GLUT3 gene, which participates in processes involved in glucose metabolism. As shown in Fig 2.

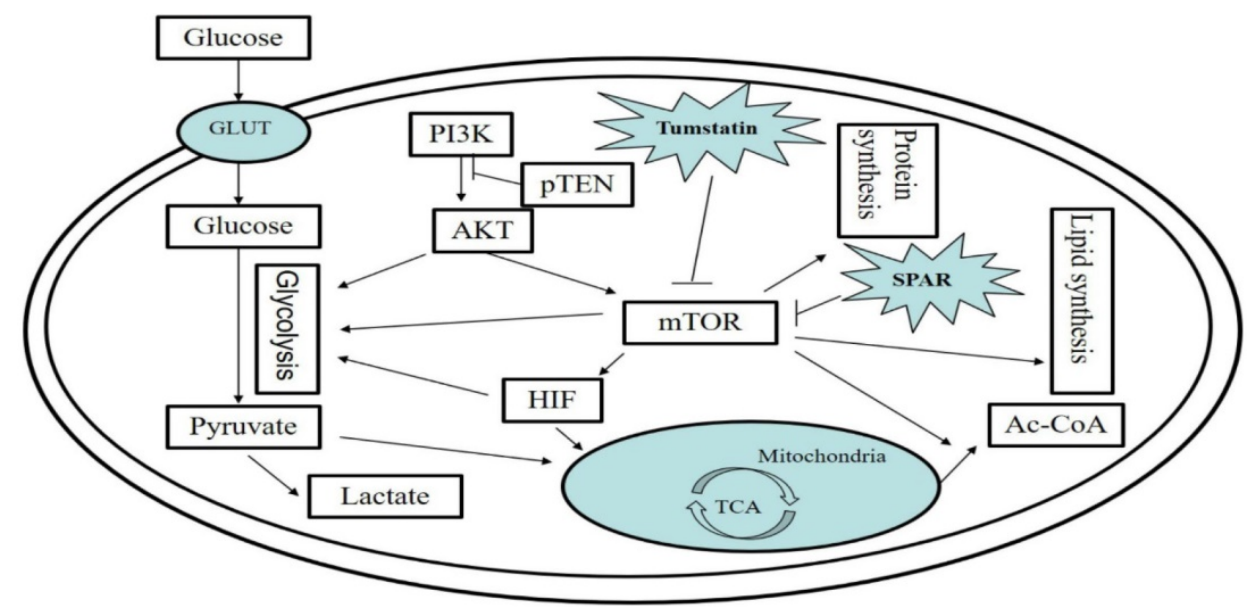

Figure 1. The PI3/AKT/mTOR signaling pathway in tumor cells and regulation by bioactive peptides of the PI3/AKT/mTOR signaling pathway. The PI3/AKT/mTOR pathway is an important signaling pathway for intracellular glucose metabolism, lipid metabolism, and protein metabolism. Small regulatory polypeptide of amino acid response (SPAR) reduced the activity of $\mathrm{mTORC}$, while tumstatin inhibited the mTOR pathway.

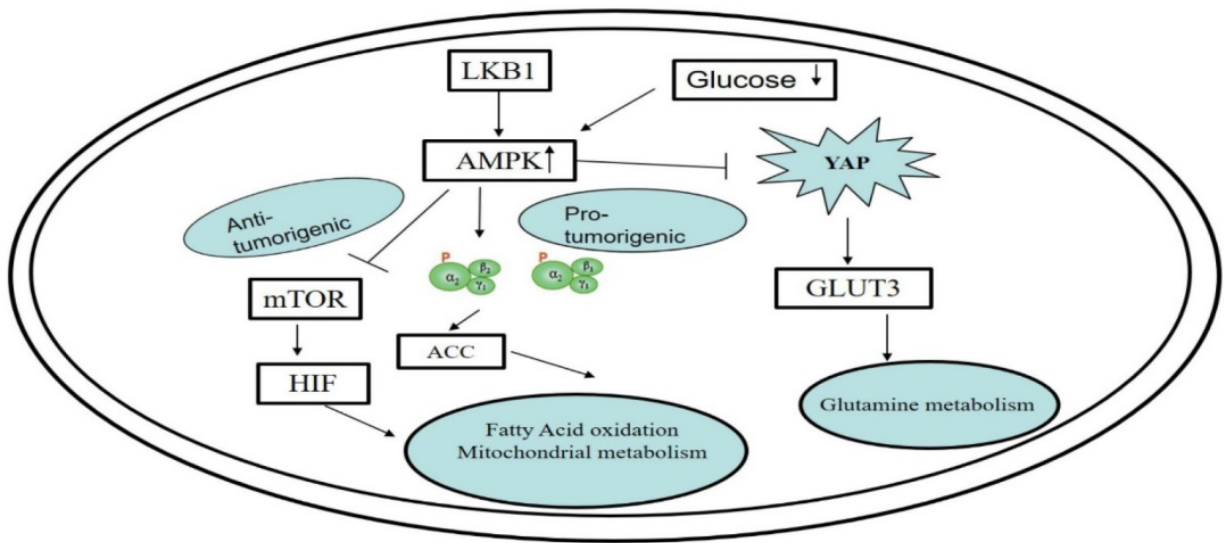

Figure 2. Functioning of the AMPK signaling pathway in cell metabolism and its regulation by bioactive peptides. The regulation of YAP can be achieved via partial manipulation of the GLUT3 gene, which participates in processes involved in glucose metabolism. 


\section{Regulation of the STAT3 signaling pathway by bioactive peptides}

STAT3 is the most ancient and active member of the STAT family. It is widely expressed in many organisms and can be activated in many ways. In addition, it participates in many biological processes, including those underlying chronic inflammation, oxidative stress, apoptosis, cell proliferation, and the restoration and regeneration of tissues. Meanwhile, it has different functions in various cells and tissues and therefore performs differently in various internal environments and disease states [30,31]. The gene encoding STAT3 is located on chromosome 12 in humans. To date, three types of STAT3 coding genes have been discovered: STAT3a, STAT3 $\beta$, and STAT3 $\gamma$. STAT3 exists in the cytoplasm and can be activated by various substances, including cytokines, growth factors, and G-CSF, and transferred into the nucleus to combine with its target gene to regulate the expression of the genes that carry out its functions [32]. Usually, STAT3 can be activated through the JAK/STAT pathway, Ras/MAPK pathway and nonreceptor tyrosine kinase pathway. The most common way STAT3 is activated is through the JAK/STAT pathway, which occurs via the following process: 1) ligands combine with receptors on the cell membrane to cause dimerization of the receptors; 2) the dimerized receptors activate JAK and facilitate its phosphorylation; 3) activated JAK facilitates phosphorylation of STAT3, which has many phosphorylation sites, including tyrosine, serine, and cysteine; the most important of these is the tyrosine 705 site that is required for the activation of STAT3 (the latest research has shown that the serine 727 is an inhibitor of downstream signal conduction, and the cysteine 259 site functions as an inhibitor of the STAT3 pathway as well); 4) the phosphorylated STAT3 dimerizes and enters the nucleus to bind with target gene promoters and thus increase their expression [33-35].

Currently, studies of the targeted inhibition of STAT3 during treatment of tumors have shown that the following methods are theoretically efficient for use in the treatment of cancer: the inhibition of protein kinases upstream of the STAT3 pathway; the inhibition of inflammation factors and receptor coupling compounds (e.g., IL-6, IL-6R/gp130 compound); inhibition of the dimerization of STAT3; blockage of nuclear translocation of STAT3; promotion of phosphatase activity upstream of the STAT3 pathway; decrease in the DNA binding ability and transcriptional activity of the STAT3 pathway $[36,37]$ Although tyrosine-phosphorylated short-peptides can greatly promote the proliferation of normal cells and reduce their apoptosis, they can hardly pass through the membrane, which restricts their wide application.
Recent studies have been focused on the use of benzoyl drugs to filter, fabricate and modify them so their inhibition efficacy and biological structureactivity can be improved to increase their ability to be effective for treatment $[38,39]$. Nagel-Wolfrum et al. filtrated a STAT3 peptide aptamer that has been shown to have specific actions using a traditional yeast two-hybrid system [40]. After it was applied it to the tumor cells, they discovered that the expression level of Bcl-xL protein was decreased within the entire STAT3 pathway, which led to the apoptosis of tumor cells. Borghouts et al. combined Western blot, gel electrophoresis and filtration with an anti-His tag to produce a specific STAT3 aptamer that inhibited signaling of STAT3 in human MZ-54 glioma cells, which resulted in the inhibition of proliferation in tumor cells [41]. As shown in Fig 3.

\section{Regulation of the TRAIL death receptor pathway by bioactive peptides}

TNF-related apoptosis inducing ligand (TRAIL), which is also known as APO2L, is a type II transmembrane protein and a member of the TNF family. TRAIL functions as immunological surveillance for anti-tumor purposes as part of the immune system, and its apoptosis-inducing c-terminal region is highly homologous with that of other TNF family members (including Fas and TNF). It is one of the important representatives of tumor cell apoptosisinducing biological drugs [42]. Usually, TRAIL acts on two kinds of receptors in the human body: TRAIL R1 and R2, which are also known as DR4 and DR5. The receptors are located on the cell membrane and can induce exogenous cell apoptosis or the death receptor pathway, which does not require the presence of p53; therefore, its antineoplastic activity is more extensive than that of therapeutic agents for DNA damage [43].

Under physiological conditions, TRAIL can act on four different transmembrane receptors in the human body: DR4, DR5, DCR1, and DCR2. TRAIL and its ligand combine to form a homogenous trimer, which is the functional form that activates APO2L when it acts on related receptors [44]. Because DCR1 lacks an intracellular domain while DCR2 has a truncated intracellular domain, they compete against one another to bind to TRAIL at similar binding sites. In addition, TRAIL can act on OPG to negatively regulate osteoclast formation and bone resorption, but the effects of TRAIL on OPG are much weaker than on other receptors. Cell apoptosis was shown to be initiated by the binding of DR4 and DR5 with ligands in the co-located signal domain of cell death [45]. Similarly, to CD95L, TRAIL has apoptotic effects but does not affect normal cells, which makes TRAIL an attractive research target for cancer therapy. 


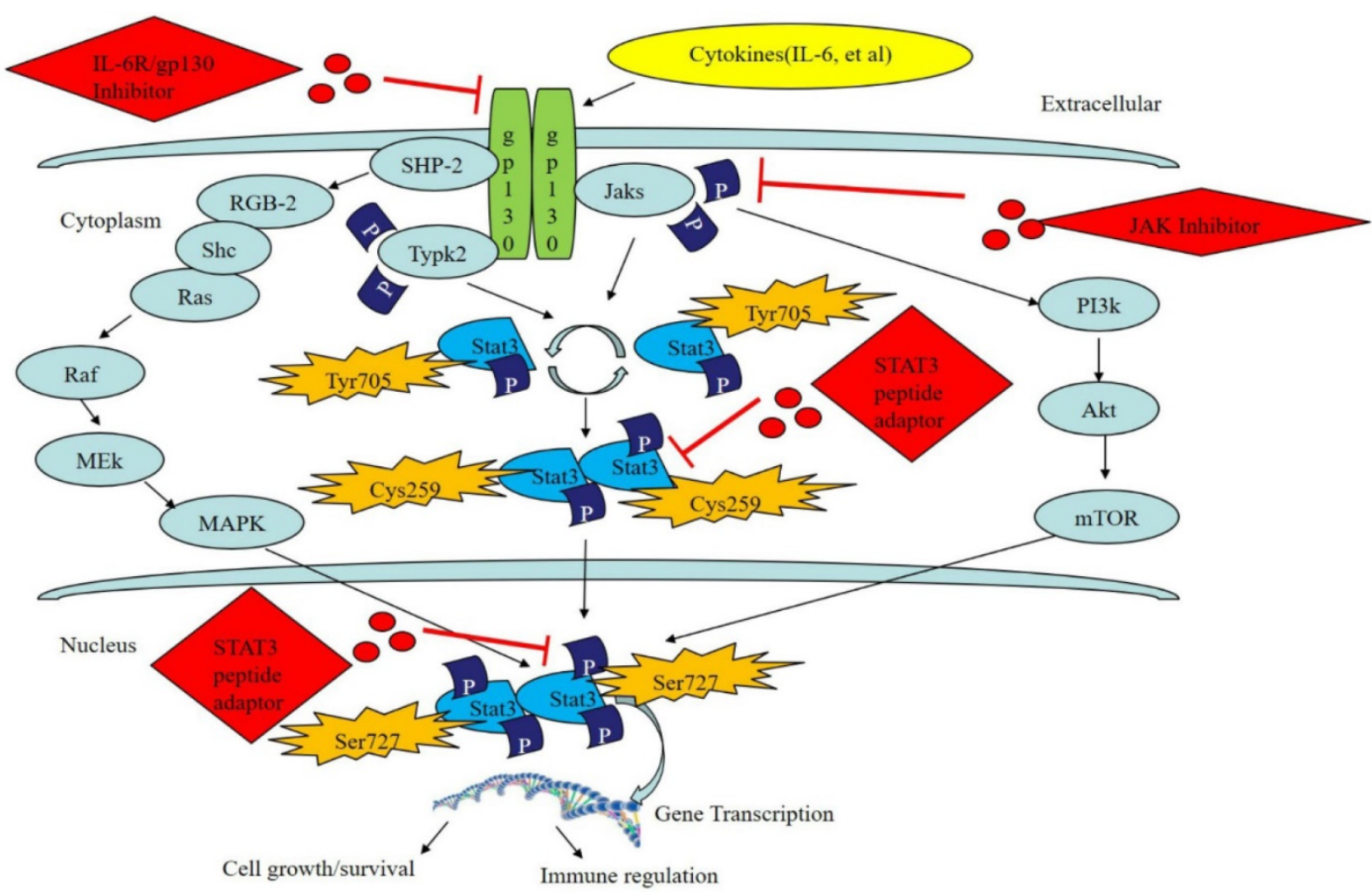

Figure 3. The STAT3 signaling pathway and its regulation by bioactive peptides. STAT3 can be activated via the JAK/STAT pathway, Ras/MAPK pathway and the nonreceptor tyrosine kinase pathway. Tyr705, Cys259 and Ser727 serve as inhibitors of the STAT3 pathway. Moreover, bioactive peptides, including the STAT3 peptide adaptor, JAK inhibitor, or IL-6R/gp130 compound inhibitor, can regulate the STAT3 signaling pathway.

Mahmood et al. found that many tumor cells resist apoptosis by developing resistance to TRAIL ligands or inhibition of the relevant signal transduction pathways, mainly by upregulating the expression of various apoptotic inhibitors, such as IAPs, anti-apoptotic Bcl-2 family protein, Caspase family proteins, c-FLIP, cytochrome $\mathrm{C}$ and Smac in mitochondria [46]. Wilson et al. used phage display technology to identify PLVAP peptides that contain sulfide structures in their conserved area, which can prevent DR5 signaling in lung cancer cells and thereby block apoptosis; however, because the DR protein combining site has several different functions, if other similar combinations of small molecular peptides can be made with DR5, it may be plausible to induce DR conformational changes and signaling pathway activation to trigger the TRAIL apoptosis signal and thereby initiate apoptosis [47]. Shen et al. applied a low dose of glutathione oxidase simulant 2-TeCD in the presence of TRAIL to kill breast cancer cells and found that 2-TeCD could moderately upregulate the expression of DR protein in the TRAIL pathway and block the activation of the NF-KB pathway, thus reducing metabolism in breast cancer cells. They also speculated that after adjusting the dose of the analog 2-TeCD, the TRAIL pathway could be sensitized by $2-\mathrm{TeCD}$ via activation of the caspase pathway and thus induce apoptosis [48]. Our previous study found that anticancer bioactive peptides (ACBPs) had the remarkable ability to inhibit human gastric cancer growth both in vitro and in vivo [49] and to sensitize tumor cells to cisplatin [50]. Furthermore, we also found that ACBPs suppress human colorectal tumor cell growth and induce apoptosis via modulation of the PARP-p53-Mcl-1 signaling pathway [51]. As shown in Fig 4.

\section{Regulation of the NF-кB signaling pathway by bioactive peptides}

As a member of the NF-KB/Rel family, NF-KB is a heterologous dimer composed of p50 and Rel A. The NF-KB signaling pathway can be found in most eukaryotes. It participates in the transcriptional regulation of many genes and is involved in tumor formation, the inflammatory cascade and apoptosis [52]. Experiments have proven that many factors can promote the activation of the NF- $\mathrm{KB}$ signal conduction pathway. Three main pathways have been found: the classical pathway, the bypass pathway and the NF-кB activation pathway, which involves precursor protein p105 [53]. Sustained activation of the NF-кB signaling pathway allows for the transcription of genes that are highly expressed in some tumor cells, while NF-KB can promote the expression of angiogenesis-related 
factors, including u-PA, VEGF, ICAM-I and MMP, and the proliferation of malignant tumor vessels [54,55]. Researchers have found that in many tumor cells, mutagenesis or abnormal degradation will occur in the presence of ІкB, while in normal cells the number of ІкB molecules does not change [56]. In addition, NF-KB can be activated by cancerous proteins and viruses in many cells and thereby induce cell carcinogenesis [57].

As for the relationship between the activity of NF-KB and tumors, the growth or proliferation of tumor cells can be blocked by inhibiting the activity of related proteins in the NF-KB signaling pathway, thereby reducing the metastasis of tumor cells and improving the therapeutic effects of tumor treatment [58]. SP is a type of bioactive peptide that is widely distributed in the central nervous system. Not only does it enhance phagocytic activity in mononuclear macrophages, promote the proliferation of $\mathrm{T}$ cells, and increase the synthesis and secretion of immunoglobulin by B cells, but it also binds to the signaling receptor NK-1 and activates MAPK to initiate the NF-KB signaling pathway [59]. Lieb et al. found that in the NF-KB signaling pathway, SP can transform the external information obtained from the binding of molecules to receptors into a signal that can be recognized inside tumor cells, which will then stimulate the expression of cell growth factor, induce DNA synthesis, and promote the proliferation and migration of tumor cells. In addition, the amount of p65 protein in cells is very small prior to their over-activation by the NF-KB pathway, which causes the massive expression of p65 and the nuclear displacement of $\mathrm{p} 65$ to serve as important markers of activation of NF-KB pathway and initiation of the NF-кB inflammatory factor pathway [60]. Shahrokhi et al. found that SP could promote the expression of p65 in tumor cells and activate the nuclear transcription factor NF-KB using a Western blot method that effectively identified p65 protein in the osteoclast tumor cells. This mechanism mainly promotes the nuclear translocation of p65 proteins. Since DNA in tumor cells and p65 have a very high affinity, p65 can serve as an efficient identifier of specific targeted tumor cells [61]. As shown in Fig 5.

\section{Conclusions}

Due to the development of metabolomics and imaging detection technology, the complex metabolic changes that are involved in the occurrence and development of tumors have become increasingly clear. The topics of research into tumor metabolism have also gradually shifted from simple glycolysis to interactions with the tumor microenvironment, tumor heterogeneity and clinical diagnosis. Metabolic regulation is not only a basic activity during tumor growth but also a powerful tool that can be used by the tumor to break through the growth barrier. The flexible and complex metabolic patterns in a tumor can not only promote tumor adaptation to different microenvironments but also contribute to the secretion of inflammatory factors that will inhibit the functioning of immune cells in the microenvironment and, thus, will interfere with normal metabolism in the body to create an environment that will promote the malignant progression of tumor cells. In addition, tumor cells share the same metabolic pathways as normal cells, which makes it difficult to intervene in processes of tumor metabolism without affecting normal cells. Therefore, there are still many difficulties remaining for the targeted treatment of tumor metabolism, and there are still many problems to be solved in the field of tumor metabolic regulation. Generally, active peptides consist of 2 to 30 amino acid residues and have beneficial features, such as strong curative effects, reduced side effects, small molecular weights, and easy absorption, that allow them to specifically bind to tumor tissues and interact with tumor growth-related and metastasis-related signal transduction molecules to inhibit tumor growth and metastasis and promote apoptosis in tumor cells. The signaling pathways involved in tumor metabolism to which bioactive peptides can be applied include PI3K/AKT/mTOR, AMPK, STAT3, TRAIL death receptor, and NF-KB signaling pathways. Based on this knowledge, polypeptide drugs designed and developed for targeted intervention in signaling pathways are designed to provide better choices for the treatment of the related clinical diseases.

At present, the application of bioactive peptides as drugs, vaccines, guiding drugs, diagnostic reagents, enzyme inhibitors and drug lead compounds has a wide theoretical basis and application value. However, there are still many challenges in the actual production and clinical application of antitumor peptides. The key to the industrialization of peptide production is the transformation of related high-tech research achievements (nanotechnology, gene engineering, enzyme engineering, etc.) in ways that promote activity protection and the use of steady-state technology for the production of anti-tumor active peptides. There are many defects in antitumor active peptides, such as frequent degradation within the body, short half-lives, unstable structures, and low bioavailability; also, macromolecular polypeptides may induce immune responses in the body, which greatly limits the clinical application of antitumor active peptides. Due to complexity in the spatial structure of peptides, their molecular characterization 
is difficult. Therefore, to further improve their application to this field, it is suggested that researchers vigorously develop all types of resources for antitumor active peptides, improve the efficiency of the preparation of antitumor active peptides, reduce their production cost using molecular modification, and utilize slow release or corresponding pseudo-peptide drug development methods to improve the stability of the peptides in the body in a way that increases their solubility and biological titer and prolongs their half-life. In addition, the mechanisms underlying the effects of antitumor active peptides from various sources and their clinical applications should be studied further.

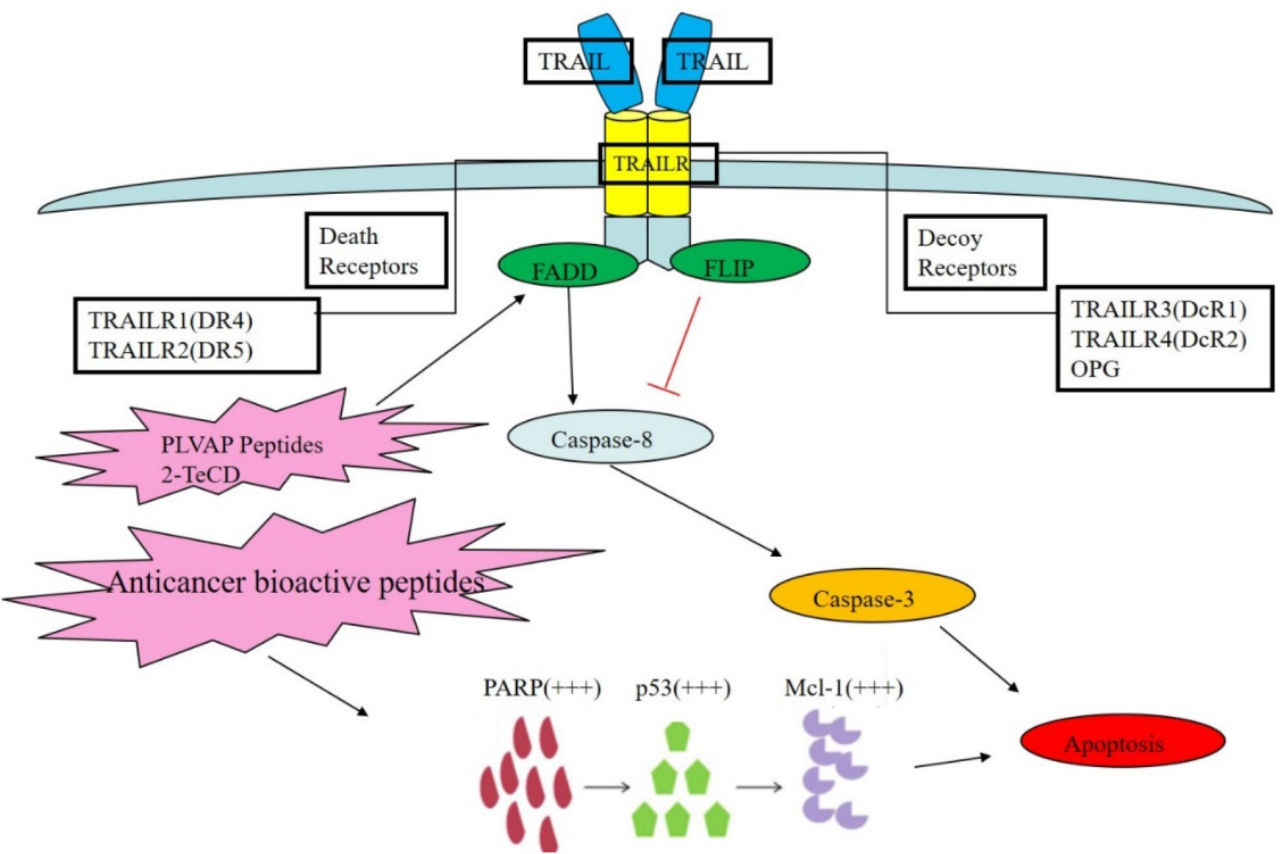

Figure 4. The TRAIL death receptor pathway and its regulation by bioactive peptides. TRAIL acts on two kinds of receptors in human body: TRAIL R1 and R2, which are also known as DR4 and DR5. Other receptors, TRAIL R3 and R4, which are known as DcR1 and DcR2, are decoy receptors for OPG. PLVAP peptides may cause DR conformational changes and signaling pathway activation, which triggers the TRAIL apoptosis signal and leads to apoptosis. Furthermore, 2-TeCD could moderately upregulate the expression of DR protein in the TRAIL pathway and, thus, inhibit metabolism in cancer cells.

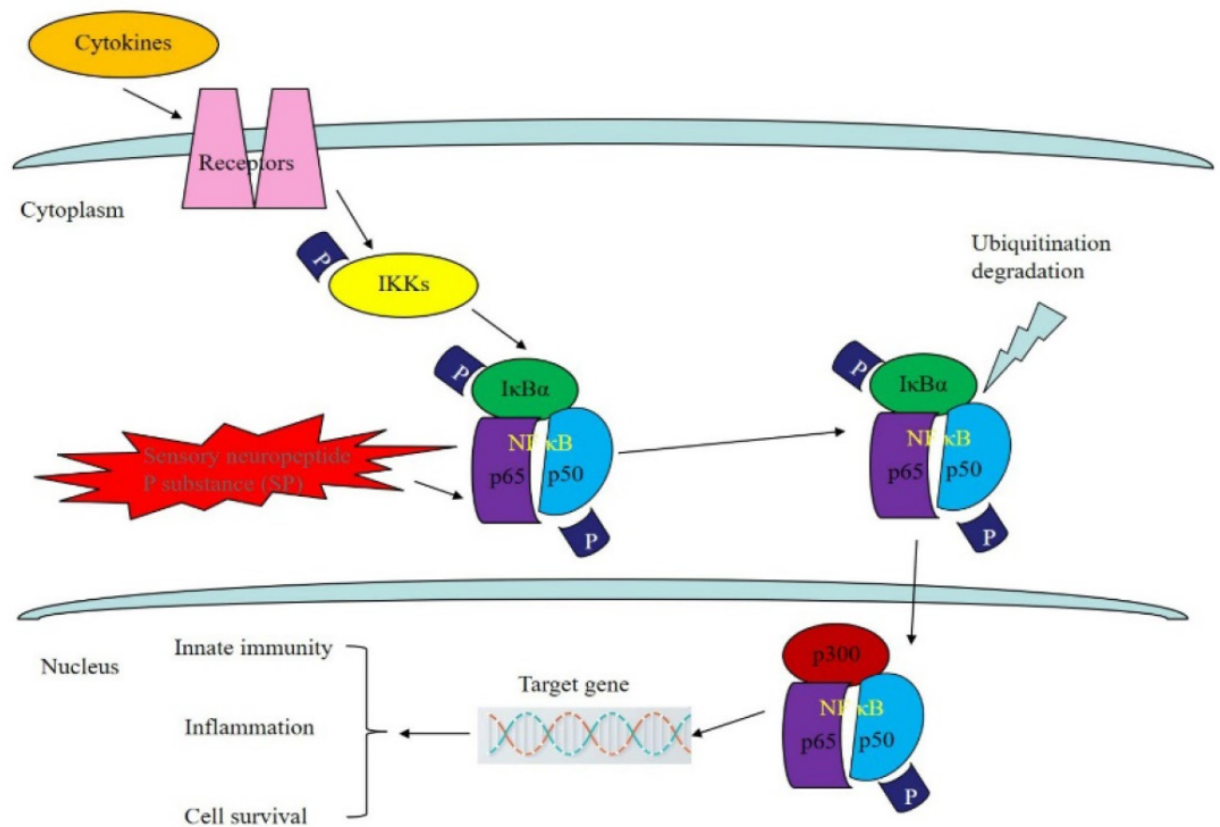

Figure 5. Regulation of NF-kB signaling pathway by bioactive peptides. As a member of the NF-kB/Rel family, NF-kB is a heterologous dimer composed of $\mathrm{p} 50$ and Rel A (p65). SP can transform the external information obtained from the binding of molecules to receptors into a signal that can be recognized inside tumor cells, which can then promote the expression of p65 in tumor cells and activate the nuclear transcription factor NF-KB. 


\section{Acknowledgments}

This research was supported in part by The National Natural Science Foundation of China(Grant Nos. 81660468); Inner Mongolia Natural Science Foundation Project of China (Grant Nos. 2017MS08 94); Inner Mongolia Autonomous Region Health and Family Planning Research Project of China (Grant Nos. 201702067).

\section{Author Contributions}

H.C., W.H. and X.S. designed the study, and wrote the review. X.S. conceived, designed the review. H.C.,J.Z. and Z.Z. edited and revised review. All authors discussed and approved the final version.

\section{Competing Interests}

The authors have declared that no competing interest exists.

\section{References}

[1] Tekade RK, Sun X. The Warburg effect and glucose-derived cancer theranostics. Drug Discov Today. 2017; 22: 1637-53.

[2] Ganapathy-Kanniappan S, Geschwind JF. Tumor glycolysis as a target for cancer therapy: progress and prospects. Mol Cancer. 2013; 12:152.

[3] Kim SY. Cancer Energy Metabolism: Shutting Power off Cancer Factory. Biomol Ther (Seoul). 2018; 26: 39-44.

[4] Muir A, Danai LV, Vander Heiden MG. Microenvironmental regulation of cancer cell metabolism: implications for experimental design and translational studies. Dis Model Mech. 2018; 11: pii: dmm035758.

[5] Boroughs LK, DeBerardinis RD. Metabolic pathways promoting cancer cell survival and growth. Nat Cell Biol.2015; 17: 351-9.

[6] Xiao YF, Jie MM, Li BS, Hu CJ, Xie R, Tang B, et al. Peptide-Based Treatment: A Promising Cancer Therapy.J Immunol Res. 2015; 2015:761820.

[7] Kaur K, Ahmed S, Soudy R, Azmi S. Screening peptide array library for the identification of cancer cell-binding peptides. Methods Mol Biol. 2015; 1248: 239-247.

[8] Min KA, Maharjan P, Ham S, Shin MC. Pro-apoptotic peptides-based cancer therapies: challenges and strategies to enhance therapeutic efficacy. Arch Pharm Res. 2018; 41: 594-616.

[9] Xing Z, Yu L, Li X, Su X. Anticancer bioactive peptide-3 inhibits human gastric cancer growth by targeting miR-338-5p. Cell Biosci. 2016; 16: 53.

[10] Yu L, Yang L, An W, Su X. Anticancer bioactive peptide-3 inhibits human gastric cancer growth by suppressing gastric cancer stem cells. J Cell Biochem. 2014; 115: 697-711.

[11] Liu Z, Fu X, Huang W, Li C, Wang X, Huang B. Photodynamic effect and mechanism study of selenium-enriched phycocyanin from Spirulina platensis against liver tumours. J Photochem Photobiol B. 2018; 180: 89-97.

[12] Courtnay R, Ngo DC, Malik N, Ververis K, Tortorella SM, Karagiannis TC. Cancer metabolism and the Warburg effect: the role of HIF-1 and PI3K. Mol Biol Rep. 2015; 42: 841-51.

[13] Dong HW, Hang LF, Bao SL. AMPK regulates energy metabolism through the SIRT1 signaling pathway to improve myocardial hypertrophy. Eur Rev Med Pharmacol Sci. 2018; 22: 2757-66.

[14] Lodi A, Saha A, Lu X, Wang B, Sentandreu E, Collins M, et al. Combinatorial treatment with natural compounds in prostate cancer inhibits prostate tumor growth and leads to key modulations of cancer cell metabolism. NPJ Precis Oncol. 2017; 1: pii: 18.

[15] Martincuks A, Fahrenkamp D, Haan S, Herrmann A, Küster A, Müller-Newen $\mathrm{G}$. Dissecting functions of the N-terminal domain and GAS-site recognition in STAT3 nuclear trafficking. Cell Signal. 2016; 28: 810-25.

[16] Mauro-Lizcano M, López-Rivas A. Glutamine metabolism regulates FLIP expression and sensitivity to TRAIL in triple-negative breast cancer cells. Cell Death Dis. 2018; 9: 205.

[17] Bi CL, Wang H, Wang YJ, Sun J, Dong JS, Meng X, et al. Selenium inhibits Staphylococcus aureus-induced inflammation by suppressing the activation of the NF-kappaB and MAPK signalling pathways in RAW264.7 macrophages. Eur J Pharmacol. 2016; 780: 159-65.

[18] Han C, Wei S, Song Q, He F, Xiong X, Wan H, et al. Insulin Stimulates Goose Liver Cell Growth by Activating PI3K-AKT-mTOR Signal Pathway. Cell Physiol Biochem. 2016: 38: 558-70.

[19] Yu JS, Cui W. Proliferation, survival and metabolism: the role of PI3K/AKT/mTOR signalling in pluripotency and cell fate determination. Development. 2016; 143: 3050-60.
[20] Yang X, Cheng Y, Li P, Tao J, Deng X, Zhang X, et al. A lentiviral sponge for miRNA-21 diminishes aerobic glycolysis in bladder cancer T24 cells via the PTEN/PI3K/AKT/mTOR axis. Tumour Biol. 2015; 36: 383-91.

[21] Xiao $Y$, Peng $H$, Hong $C$, Chen $Z$, Deng $X$, Wang A, et al. PDGF Promotes the Warburg Effect in Pulmonary Arterial Smooth Muscle Cells via Activation of the PI3K/AKT/mTOR/HIF-1alpha Signaling Pathway. Cell Physiol Biochem. 2017; 42: 1603-13.

[22] Pusapati RV, Daemen A, Wilson C, Sandoval W, Gao M, Haley B, et al. mTORC1-Dependent Metabolic Reprogramming Underlies Escape from Glycolysis Addiction in Cancer Cells. Cancer Cell. 2016; 29: 548-62.

[23] Matsumoto A, Pasut A, Matsumoto M, Yamashita R, Fung J, Monteleone E, et al . mTORC1 and muscle regeneration are regulated by the LINC00961-encoded SPAR polypeptide. Nature.2017; 541: 228-32.

[24] Li T, Kang G, Wang T, Huang H. Tumor angiogenesis and anti-angiogenic gene therapy for cancer. Oncol Lett. 2018; 16: 687-702.

[25] Voss CM, Pajęcka K, Stridh MH, Nissen JD, Schousboe A, Waagepetersen HS. AMPK Activation Affects Glutamate Metabolism in Astrocytes. Neurochem Res. 2015; 40: 2431-42.

[26] Wu B, Chen X, Zhou Y, Hu P, Wu D, Zheng G, et al. Andrographolide inhibits proliferation and induces apoptosis of nasopharyngeal carcinoma cell line C666-1 through LKB1-AMPK-dependent signaling pathways. Pharmazie. 2018; 73: 594-7.

[27] Zhou W, Zhang J, Marcus AI. LKB1 Tumor Suppressor: Therapeutic Opportunities Knock when LKB1 Is Inactivated. Genes Dis. 2014; 1: 64-74.

[28] Wang W, Xiao ZD, Li X, Aziz KE, Gan B, Johnson RL, et al. AMPK modulates Hippo pathway activity to regulate energy homeostasis. Nat Cell Biol. 2015; 17: 490-9.

[29] Mo JS, Meng Z, Kim YC, Park HW, Hansen CG, Kim S, et al. Cellular energy stress induces AMPK-mediated regulation of YAP and the Hippo pathway. Nat Cell Biol. 2015; 17: 500-10.

[30] Laudisi F, Cherubini F, Monteleone G, Stolfi C. STAT3 Interactors as Potential Therapeutic Targets for Cancer Treatment. Int J Mol Sci. 2018; 19: pii: E1787.

[31] Fathi N, Rashidi G, Khodadadi A, Shahi S, Sharifi S. STAT3 and apoptosis challenges in cancer. Int J Biol Macromol. 2018; 117: 993-1001.

[32] Wake MS, Watson CJ. STAT3 the oncogene-still eluding therapy?. FEBS J. 2015; 282: 2600-11.

[33] Qi QR, Yang ZM. Regulation and function of signal transducer and activator of transcription 3. World J Biol Chem.2014; 5: 231-9.

[34] Wu J, Du J, Fu X, Liu B, Cao H, Li T, et al. A novel FASN inhibitor, exerts anti-melanoma activities through IGF-1R/STAT3 signaling. Oncotarget. 2016; 7: 51251-69.

[35] Kim J, Won JS, Singh AK, Sharma AK, Singh I. STAT3 regulation by S-nitrosylation: implication for inflammatory disease. Antioxid Redox Signal. 2014; 20: 2514-27.

[36] Kuang S, Qi C, Liu J, Sun X, Zhang Q, Sima Z, et al. 2-Methoxystypandrone inhibits signal transducer and activator of transcription 3 and nuclear factor-kappaB signaling by inhibiting Janus kinase 2 and IkappaB kinase. Cancer Sci. 2014: 105: 473-80.

[37] Nelson EA, Sharma SV, Settleman J, Frank DA. A chemical biology approach to developing STAT inhibitors: molecular strategies for accelerating clinical translation. Oncotarget. 2011; 2: 518-24.

[38] Fletcher S, Turkson J, Gunning PT. Molecular approaches towards the inhibition of the signal transducer and activator of transcription 3 (Stat3) protein. ChemMedChem.2008; 3: 1159-68.

[39] Turkson J, Ryan D, Kim JS, Zhang Y, Chen Z, Haura E, et al. Phosphotyrosyl peptides block Stat3-mediated DNA binding activity, gene regulation, and cell transformation. J Biol Chem. 2001; 276: 45443-55.

[40] Nagel-Wolfrum K, Buerger C, Wittig I, Butz K, Hoppe-Seyler F, Groner B. The interaction of specific peptide aptamers with the DNA binding domain and the dimerization domain of the transcription factor Stat3 inhibits transactivation and induces apoptosis in tumor cells. Mol Cancer Res. 2004; 2: $170-82$.

[41] Borghouts C, Kunz C, Delis N, Groner B. Monomeric recombinant peptide aptamers are required for efficient intracellular uptake and target inhibition. Mol Cancer Res. 2008; 6: 267-81.

[42] Guicciardi ME, Mott JL, Bronk SF, Kurita S, Fingas CD, Gores GJ. Cellular inhibitor of apoptosis 1 (cIAP-1) degradation by caspase 8 during TNF-related apoptosis-inducing ligand (TRAIL)-induced apoptosis. Exp Cell Res. 2011; 317: 107-16

[43] van-Roosmalen IA, Quax WJ, Kruyt FA.Two death-inducing human TRAIL receptors to target in cancer: similar or distinct regulation and function? Biochem Pharmacol. 2014; 91: 447-56.

[44] Allen JE, El-Deiry WS. Regulation of the human TRAIL gene. Cancer Biol Ther. 2012; 13: 1143-51.

[45] Sarhan D, D'Arcy P, Lundqvist A. Regulation of TRAIL-receptor expression by the ubiquitin-proteasome system. Int J Mol Sci. 2014; 15: 18557-73.

[46] Mahmood Z, Shukla Y. Death receptors: targets for cancer therapy. Exp Cell Res. 2010; 316: 887-99.

[47] Wilson NS, Yang A, Yang B, Couto S, Stern H, Gogineni A, et al. Proapoptotic activation of death receptor 5 on tumor endothelial cells disrupts the vasculature and reduces tumor growth. Cancer Cell. 2012; 22: 80-90.

[48] Lin T, Ding Z, Li N, Xu J, Luo G, Liu J, et al. 2-Tellurium-bridged beta-cyclodextrin, a thioredoxin reductase inhibitor, sensitizes human breast cancer cells to TRAIL-induced apoptosis through DR5 induction and NF-kappaB suppression. Carcinogenesis. 2011; 32: 154-67. 
[49] Su LY, Xin HY, Liu YL, Zhang JL, Xin HW, Su XL. Anticancer bioactive peptide (ACBP) inhibits gastric cancer cells by upregulating growth arrest and DNA damage-inducible gene 45A (GADD45A). Tumour Biol. 2014; 35:10051-56.

[50] Su X, Dong C, Zhang J, Su L, Wang X, Cui H, et al. Combination therapy of anti-cancer bioactive peptide with Cisplatin decreases c.hemotherapy dosing and toxicity to improve the quality of life in xenograft nude mice bearing human gastric cancer. Cell Biosci. 2014; 4:7.

[51] Su LY, Shi YX, Yan MR, Xi Y, Su XL. Anticancer bioactive peptides suppress human colorectal tumor cell growth and induce apoptosis via modulating the PARP-p53-Mcl-1 signaling pathway. Acta Pharmacol Sin. 2015; 36: 1514-19.

[52] Vallabhapurapu S, Karin M. Regulation and function of NF-kappaB transcription factors in the immune system. Annu Rev Immunol. 2009; 27: 693-733.

[53] Bonizzi G, Karin M. The two NF-kappaB activation pathways and their role in innate and adaptive immunity. Trends Immunol.2004; 25: 280-8.

[54] Yang X, Shi B, Li L, Xu Z, Ge Y, Shi J, et al. Death receptor 6 (DR6) is required for mouse B16 tumor angiogenesis via the NF-kappaB, P38 MAPK and STAT3 pathways. Oncogenesis. 2016; 5: e206.

[55] Chen Z, Li Z, Chang Y, Ma L, Xu W, Li M, et al. Relationship between NF-kappaB, MMP-9, and MICA expression in pituitary adenomas reveals a new mechanism of pituitary adenomas immune escape. Neurosci Lett. 2015; 597: 77-83.

[56] Willems M, Dubois N, Musumeci L, Bours V, Robe PA. IkappaBzeta: an emerging player in cancer. Oncotarget. 2016; 7: 66310-22.

[57] Shostak K, Chariot A. EGFR and NF-kappaB: partners in cancer. Trends Mol Med. 2015; 21: 385-93.

[58] Sawant RR, Jhaveri AM, Koshkaryev A, Qureshi F, orchilin VP. The effect of dual ligand-targeted micelles on the delivery and efficacy of poorly soluble drug for cancer therapy. J Drug Target. 2013; 21: 630-8.

[59] Kong L, Liu J, Wang J, Luo O, Zhang H, Liu B, et al. Icariin inhibits TNF-alpha/IFN-gamma induced inflammatory response via inhibition of the substance $\mathrm{P}$ and $\mathrm{p} 38$-MAPK signaling pathway in human keratinocytes. Int Immunopharmacol. 2015; 29: 401-7.

[60] Lieb K, Fiebich BL, Berger M, Bauer J, Schulze-Osthoff K. The neuropeptide substance $\mathrm{P}$ activates transcription factor NF-kappa B and kappa B-dependent gene expression in human astrocytoma cells. J Immunol. 1997; 159: 4952-8.

[61] Shahrokhi S, Ebtekar M, Alimoghaddam K, Pourfathollah AA, Kheirandish M, Ardjmand A, et al. Substance P and calcitonin gene-related neuropeptides as novel growth factors for ex vivo expansion of cord blood CD34(+) hematopoietic stem cells. Growth Factors. 2010; 28: 66-73. 